\title{
Leucemia eosinofílica crônica com expressão do rearranjo FIP1L1-PDGFR $\alpha$ - relato de caso e revisão da literatura
}

Chronic eosinophilic leukemia with a FIP1L1-PDGFR $\alpha$ fusion-case report and literature review

Martha M. A. S. Arruda

Alex F. Sandes ${ }^{2}$

Natalia M. Borges ${ }^{3}$

Maria de Lourdes L. F. Chauffaille
A eosinofilia no sangue e em tecidos está habitualmente associada a condições alérgicas, infecciosas, inflamatórias, neoplásicas, endocrinológicas, uso de medicamentos e exposição a agentes tóxicos. No entanto, pode ocorrer proliferação eosinofilica primária, sem causa aparente ou por expansão clonal da célula-tronco hematopoética. As neoplasias mielo ou linfoproliferativas associadas a rearranjos gênicos como PDGFR $\alpha$, PDGFR $\beta$ e FGFR1 constituem condições raras nas quais ocorre mieloproliferação crônica, alterações no sangue periférico e na medula óssea e lesão tecidual de diferentes órgãos, a partir da liberação de citocinas e fatores humorais pelos grânulos eosinofilicos. A presença do rearranjo PDGFR $\alpha$ relaciona-se comumente à leucemia eosinofilica crônica, com envolvimento de mastócitos e neutrófilos e, mais raramente, à leucemia mieloide aguda ou ao linfoma linfoblástico $T$, com eosinofilia. O rearranjo PDGFR $\alpha$ mais comum é aquele resultante da deleção intersticial no braço longo do cromossomo 4, que permite a formação de um neogene a partir da fusão dos genes FIP1L1 e PDGFR $\alpha$. Este codifica uma tirosino-quinase constitutivamente ativa que é inibida pelo mesilato de imatinibe. Em 2002 foi relatado o uso bem sucedido de mesilato de imatinibe em baixas doses em um paciente com sindrome hipereosinofilica e, desde então, vem-se utilizando esta droga com respostas clínicas rápidas, completas e duradouras. Descrevemos um caso de LEC com expressão do rearranjo FIP1L1PDGFRo. Rev. Bras. Hematol. Hemoter. 2010;32(2):177-180.

Palavras-chave: Eosinofilia; rearranjo gênico; hibridização in situ fluorescente; síndrome hipereosinofilica; leucemia.

\section{Introdução}

A eosinofilia no sangue e em tecidos está habitualmente associada a condições alérgicas, infecciosas, inflamatórias, neoplásicas, endocrinológicas, uso de medicamentos e exposição a tóxicos. ${ }^{1}$ Nessas situações, o fenômeno é desencadeado por perda da regulação ou estímulo da produção de citocinas inflamatórias. ${ }^{2}$ No entanto, pode ocorrer proliferação eosinofílica primária, sem causa aparente, ou por expansão clonal da célula-tronco hematopoética. Leucemia eosinofílica crônica (LEC) é caracterizada quando se observa evidência de expansão clonal, inclusive com aumento do número de mieloblastos. ${ }^{3}$ A presença de eosinofilia na ausência de fator desencadeante e sem evidência de clonalidade em paciente com lesão orgânica é denominada síndrome hipereosinofílica (SHE). ${ }^{3}$

${ }^{I}$ Residência médica em Hematologia e Hemoterapia pela Universidade Federal de São Paulo (EPM/Unifesp) - São Paulo-SP.

${ }_{3}^{2}$ Residência em Hematologia e Hemoterapia no Hospital do Servidor Público Municipal de São Paulo - São Paulo-SP.

${ }_{3}^{3}$ Médica residente em Clínica Médica no Hospital Guilherme Álvaro e em Hematologia e Hemoterapia pela Unifesp - São Paulo-SP.

${ }^{4}$ Professora livre docente da Disciplina de Hematologia e Hemoterapia da Unifesp - São Paulo-SP.

Disciplina de Hematologia e Hemoterapia, Universidade Federal de São Paulo - EPM/Unifesp - São Paulo-SP.

Correspondência: Maria de Lourdes Chauffaille

Disciplina de Hematologia e Hemoterapia. Escola Paulista de Medicina, Unifesp

Rua Pedro de Toledo, 650, $3^{\circ}$ Andar

04039-032 - São Paulo-SP - Brasil

Tel.: (55 11) 5576-4240; fax: (55 11) 5571-8806

E-mail: chauffaill@hemato.epm.br

Doi: 10.1590/S1516-84842010005000038 
As neoplasias mielo ou linfoproliferativas associadas a rearranjos gênicos como PDGFR $\alpha$, PDGFRe FGFR1 são condições raras que geram doenças com características semelhantes, tais como mieloproliferação crônica e ocasionalmente linfoproliferação. A presença do rearranjo PDGFR $\alpha$ relaciona-se comumente à LEC com envolvimento de mastócitos e neutrófilos e, mais raramente, à leucemia mieloide aguda (LMA) ou ao linfoma linfoblástico (LLA) T com eosinofilia. ${ }^{4}$

Clinicamente, os portadores de rearranjos envolvendo o PDGFR $\alpha$ apresentam alterações no sangue e na medula óssea (MO) e lesão de diferentes órgãos, induzidas pela liberação de citocinas e fatores humorais dos grânulos eosinofílicos. Queixas como fadiga e prurido são frequentes, assim como esplenomegalia. Podem ocorrer alterações gastrointestinais e cardíacas, sendo a endomiocardiofibrose a complicação mais grave.

O rearranjo PDGFR $\alpha$ mais comum é aquele resultante da deleção intersticial no braço longo do cromossomo 4, região na qual se localiza o gene $\mathrm{CHIC}$, que permite a formação de um neogene a partir da fusão dos genes FIP1L1 e PDGFR $\alpha .{ }^{5}$ Este codifica uma tirosina quinase constitutivamente ativa que, assim como a proteína gerada pela fusão BCR-ABL, é inibida pelo mesilato de imatinibe. ${ }^{6}$

Antes da descrição da anormalidade molecular, o tratamento das eosinofilias primárias e idiopáticas (EPI) incluía interferon- $\alpha$, hidroxiureia, alquilantes, 2 -clorodesoxiadenosina, corticoesteroides e imunossupressores. No entanto, essas abordagens não geravam resposta satisfatória ou duradoura, e causavam diversos efeitos colaterais. ${ }^{7}$ Em 2002 foi relatado tratamento bem sucedido de um caso de SHE com baixas doses de imatinibe ${ }^{8}$ e atualmente seu uso foi descrito em mais de 150 pacientes, ${ }^{7,9}$ com resposta clínica rápida, completa e duradoura. ${ }^{10,11}$

Descrevemos um caso de LEC com expressão do rearranjo FIP1L1-PDGFR $\alpha$.

\section{Relato de Caso}

Homem de 44 anos com dor leve contínua em hipocôndrio esquerdo, não aliviada por analgésicos comuns, associada à queimação epigástrica importante, sem melhora após medidas higiênico-dietéticas e antiácidos, há 2 anos. Há 8 meses apresenta lesões úlcero-crostosas, eritematosas em lábios e ulceradas em mucosa jugal, dolorosas, sangrantes (Figura 1) e máculas eritematosas que evoluíram rapidamente para lesões úlcero-crostosas, eritematosas, não pruriginosas, não dolorosas, não sangrantes, difusas em face, pescoço e membros superiores, piores em áreas expostas ao sol (Figura 2). Encaminhado ao Ambulatório de Hematologia da Unifesp por esplenomegalia e eosinofilia há mais de seis meses $\left(9 \times 10^{9} / \mathrm{L}\right)$. Nenhum antecedente familiar, social, patológico ou laboral relevante, sem história de alergias ou atopia. Exame físico: normocorado, baço palpável a $5 \mathrm{~cm}$ da borda costal

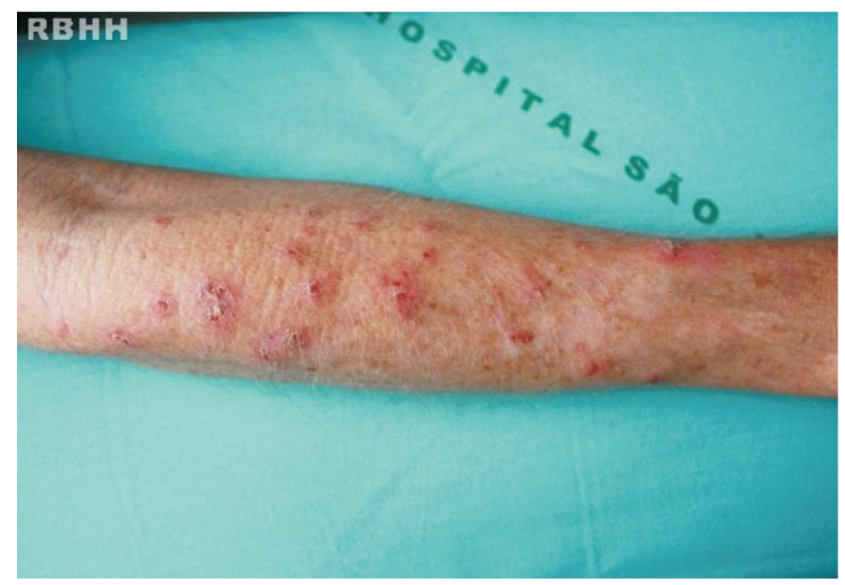

Figura 1. Lesões de pele

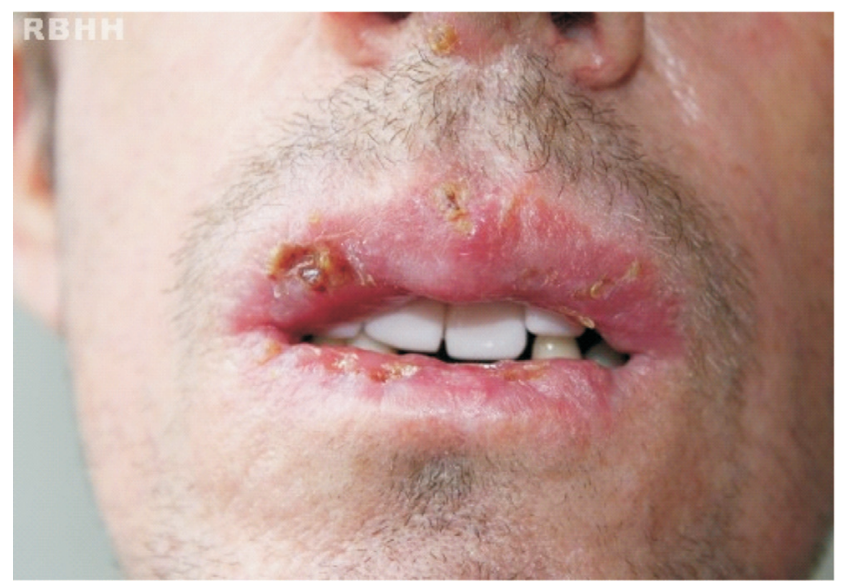

Figura 1. Lesões de mucosa

esquerda, ausência de linfonodomegalias e lesões mucocutâneas já descritas. Hemograma: hemoglobina $10 \mathrm{mg} / \mathrm{dL}$, leucócitos $18,2 \times 10^{9} / \mathrm{L}$, eosinófilos $9,1 \times 10^{9} / \mathrm{L}$ (sem anormalidades morfológicas), plaquetas $177 \times 10^{9} / \mathrm{L}$. Desidrogenase láctica, ferritina, albumina, vitamina B12 e IgE séricas normais. Sorologias para HIV e hepatites virais, provas reumatológicas e inflamatórias negativas. Pesquisas de ovos e parasitas nas fezes negativas, com persistência da eosinofilia após cursos de albendazol e ivermectina. Ecodopplercardiograma com espessamento mitral, ultrassonografia abdominal com esplenomegalia homogênea $(16 \mathrm{~cm})$ e tomografias (tórax/abdome) sem achados relevantes. Endoscopia digestiva demonstrou gastrite enantematosa leve e biópsia de mucosa gástrica com gastrite crônica moderada, sem evidência de infecção pelo Helicobacter pilory. Biópsia de pele com ectasia vascular inespecífica e biópsia de mucosa jugal com ulceração mucosa e infiltrado coriônico difuso por mononucleares, neutrófilos e eosinófilos. Aspirado MO: hipercelular, relação G/E: 5/1, série granulocítica hipercelular, com escalonamento maturativo preservado (blastos $2 \%$ ) e presença de eosinofilia (30\%). Biópsia MO: celularidade $100 \%, 90 \%$ da qual repre- 
sentada por eosinófilos e precursores. Cariótipo MO (banda G): 46, XY em 20 metáfases. Pesquisa BCR-ABL (RT-PCR): negativa. Hibridação in situ por fluorescência (FISH) para anormalidade de CHIC2, testado com sonda 4q12 (FIP1L1, CHIC2, PDGFR $\alpha$ ): $85.5 \%$ núcleos com um sinal CHIC2, dois sinais FIP1L1 e PDGFR $\alpha$ em 200 células.

Vinha em uso de hidroxiureia há dois anos, sem melhora das queixas, e com anemia. Iniciado tratamento com imatinibe $100 \mathrm{mg} /$ dia com resolução das dermatoses das queixas gastrointestinais após 15 dias e desaparecimento dos sinais de esplenomegalia após 30 dias de tratamento. Hemograma após 30 dias: hemoglobina $10 \mathrm{mg} / \mathrm{dL}$, leucócitos $2,93 \times 10^{9} / \mathrm{L}$, eosinófilos $1,55 \times 10^{9} / \mathrm{L}$, plaquetas $247 \times 10^{9} / \mathrm{L}$. Após três meses: hemoglobina $14.8 \mathrm{mg} / \mathrm{dL}$, leucócitos $5,75 \times 10^{9} / \mathrm{L}$, eosinófilos $2,34 \times 10^{9} / \mathrm{L}$, plaquetas $247 \times 10^{9} / \mathrm{L}$.

\section{Discussão}

Síndromes de EPI são, por definição, condições de eosinofilia persistente $\left(>1,5 \times 10^{9} / \mathrm{L}\right.$ por seis meses $) \mathrm{com}$ evidência de lesão orgânica. ${ }^{12}$ São doenças raras, com incidência de 0.5-1/100.000/ano ${ }^{4}$ e maior prevalência em caucasianos, principalmente homens (H:M 9:1 na SHE7 e 17:1 nas anormalidades PDGFR $\alpha)^{4}$ e pico de incidência na quarta década. O diagnóstico requer exclusão de eosinofilia reacional, notadamente infecções parasitárias, alergias, doenças pulmonares, síndrome de Loeffler, eosinofilia cíclica, doenças de pele como hiperplasia angiolinfoide, colagenoses, vasculites, doenças granulomatosas, síndrome de Kimura, linfomas de células-T, linfoma de Hodgkin, mastocitose sistêmica, LLA, LMA, leucemia mieloide crônica e outras neoplasias mieloides associadas com produção anormal de interleucina (IL) 2, IL-3, IL-5 e G-CSF. ${ }^{3}$

A alteração sanguínea mais importante é a eosinofilia, com células em sua maioria maduras, eventualmente com granulação esparsa e irregular, segmentação nuclear aberrante e aumento no tamanho. ${ }^{13} \mathrm{MO}$ apresenta hipercelularidade por aumento da proliferação eosinofílica, setores eritroide e megacariocítico normais. Pode-se observar aumento do número de mieloblastos (2\%-19\%) e fibrose medular na LEC. ${ }^{3}$ Apesar da falta de anormalidades imunofenotípicas específicas de EPI, ${ }^{3}$ a citometria de fluxo possui utilidade no diagnóstico diferencial de eosinofilia, podendo identificar casos com população clonal de linfócitos-T e detectar pacientes com mastocitose sistêmica, através da expressão anômala de CD2 e CD25 em mastócitos. ${ }^{13} \mathrm{Na}$ maioria dos casos o cariótipo é normal, inclusive nas neoplasias com anormalidade PDGFR $\alpha$, uma vez que a $\operatorname{del}(4 q 12)$ não é detectável por citogenética clássica. ${ }^{7,9}$ No entanto, a deteç̧ão de anormalidade cariotípica recorrente usualmente observada em neoplasias mieloides (e.g., i(17q), +8) permite diagnóstico de LEC. ${ }^{3,13}$ Pode-se ainda utilizar análise do polimorfismo ligado ao X dos genes PGK ou Humara em mulheres, na tentativa de demonstrar clonalidade.,13
A apresentação clínica é variável, desde diagnóstico incidental $(12 \%)$ a prurido, sintomas constitucionais e relacionados à lesão multissistêmica. ${ }^{3}$ Esplenomegalia ocorre em 30\%-50\% das LEC e, na maioria das anormalidades PDGRF $\alpha$ e observa-se hepatomegalia ocasionalmente. Infiltração tecidual e liberação de citocinas, enzimas e fatores humorais dos grânulos eosinofílicos lesam especialmente coração, pulmões, pele, sistema nervoso central e periférico, e gastrointestinal. ${ }^{3,4,7,9,14}$

A maior causa de morbimortalidade é a cardiopatia, que acontece em até $50 \%$ dos casos. ${ }^{7}$ Ocorre endomiocardiofibrose, que evolui para cardiomiopatia restritiva, além de espessamento e fibrose das valvas atrioventriculares, com regurgitação e formação de trombos intracardíacos. ${ }^{4} \mathrm{O}$ uso de imatinibe em pacientes com fibrose avançada e fusão FIP1L1-PDGRF $\alpha$ não melhora o desempenho cardíaco, independente da resposta hematológica. ${ }^{7,9}$ Isto pontua a necessidade de diagnóstico precoce e pronta intervenção.

Manifestações cutâneas ocorrem em mais de 50\% dos casos, sendo mais frequentes na fusão FIP1L1-PDGRF $\alpha .{ }^{15}$ Observam-se lesões angioedematosas, urticariformes, eritematosas, pruriginosas com pápulas e nódulos, úlceras mucosas. ${ }^{15}$ Histologicamente nota-se infiltração perivascular eosinofílica, neutrofílica e mononuclear. Todos os casos descritos com fusão FIP1L1-PDGRF $\alpha$ e acometimento mucocutâneo submetidos a tratamento com imatinibe tiveram desaparecimento das lesões cutâneas, de maneira sustentada. $^{7}$

O único ensaio prospectivo que avaliou o uso de imatinibe em EPI estudou 63 pacientes, encontrando rearranjo FIP1L1-PDGRF $\alpha$ em 27 (43\%). Estes eram todos homens, e apresentavam menor idade, maior número de eosinófilos do que os pacientes sem o rearranjo e apenas eles apresentavam lesões cutâneas. Resposta hematológica completa (RHC) foi atingida em um mês e resposta molecular completa (RMC) em dez meses de tratamento com doses entre $100-400 \mathrm{mg} / \mathrm{d}$. Todos os casos mantiveram a resposta durante o acompanhamento (15-60 meses, mediana 25 meses). Três pacientes descontinuaram o tratamento após um ano, com detecção de transcritos por RT-PCR seis meses após a interrupção e apresentaram RMC após o reinício da droga. Dentre os pacientes sem fusão, $13 \%$ apresentaram RHC em três meses; porém, todos recaíram em até 15 meses. ${ }^{9}$ Observou-se toxicidade hematológica leve (8\% grau-II, 3\%-III), e não hematológica limitada (mialgia 10\%-II, diarreia 6\%-II, edema periférico 3\%II), com excelentes índices de adesão ao tratamento. ${ }^{9}$ Acreditase que doses de imatinibe entre 100 e $200 \mathrm{mg} / \mathrm{d}$ sejam suficientes. Atualmente, levando-se em consideração o custo da droga, que pode limitar o acesso a ela, investiga-se a possibilidade de uso de doses ainda menores, inclusive semanais. ${ }^{14,16}$

Considerando que pacientes com eosinofilia e fusão FIP1L1-PDGRF $\alpha$ apresentam altas taxas de resposta clínica, hematológica e molecular e boa tolerância ao tratamento 
crônico com baixas doses de imatinibe, ${ }^{9}$ todos os pacientes com eosinofilia, descartadas as causas secundárias, devem ser prontamente submetidos à pesquisa da fusão FIP1L1PDGRF $\alpha$. Esta pesquisa pode ser realizada por FISH ou RTPCR, no sentido de abreviar tempo e recursos gastos com outras investigações e reduzir o risco de lesões orgânicas irreversíveis.

\section{Abstract}

Chronic eosinophilia is habitually associated with allergic, infectious, inflammatory, neoplastic and endocrine conditions and exposure to certain drugs and toxic agents. However, eosinophilic proliferation may be primary, without identifiable causes, or provoked by clonal hematopoietic stem cell proliferation. Gene fusions involving PDGFR- $\alpha, P D G F R-\beta$, and FGFR1 predispose patients to rare conditions with chronic myeloproliferation or lymphoproliferation, alterations in peripheral blood and bone marrow and diffuse tissue injury due to the release of cytokines and humoral factors from eosinophilic granules. The presence of the PDGFR- $\alpha$ rearrangement is commonly related to chronic eosinophilic leukemia, with alterations in peripheral mastocytes and neutrophils, and rarely to acute myeloid leukemia or $T$ lymphoblastic lymphoma with eosinophilia. The most prevalent PDGFR- $\alpha$ rearrangement is one resulting from an interstitial deletion in the long arm of chromosome 4, that allows the formation of a neogene from the fusion of the FIP1L1 and PDGFR- $\alpha$ genes. This codes a constitutively active tyrosine kinase, which can be inhibited by imatinib mesylate. In 2002, the successful treatment of a patient using imatinib to treat hypereosinophilic syndrome was reported. Since then, this drug has been utilized with fast, complete and lasting clinical responses. Here we describe a case of chronic eosinophilic syndrome with expression of the FIP1L1-PDGFR- $\alpha$ rearrangement. Rev. Bras. Hematol. Hemoter. 2010;32(2):177180.

Key words: Eosinophilia ; gene rearrangement; in situ hybridization, fluorescence; hypereosinophilic syndrome; leukemia.

\section{Referências Bibliográficas}

1. Sheikh J, Weller PF. Advances in diagnosis and treatment of eosinophilia. Curr Opin Hematol. 2009;16(1)3-8.

2. Tefferi A, Patnaik MM, Pardanani A. Eosinophilia: secondary, clonal and idiopathic. Br J Haematol. 2006.133(5):468-92.

3. Bain B, Gilliland DG, Vardiman JW, Horny H-P. Chronic eosinophilic leukaemia, not otherwise specified, inWHO Classification of Tumour of Haematopoietic and Lymphoid Tissues, Swerdlow SH, et al, Editors. 2008: Lion. p. 51-53.

4. Bain B, Gilliland DG, Vardiman JW, Horny H-P. Myeloid and lymphoid neoplasms with eosinophilia and abnormalities of PDGFRA, PDGFRB or FGFR1, in WHO Classification of Tumour of Haematopoietic and Lymphoid Tissues, Swerdlow SH, et al., Editors. 2008: Lion. p. 68-73.

5. Cools J, et al. A tyrosine kinase created by fusion of the PDGFRA and FIP1L1 genes as a therapeutic target of imatinib in idiopathic hypereosinophilic syndrome. N Engl J Med. 2003; 348(13):1201-14.
6. Carroll M, et al. CGP 57148, a tyrosine kinase inhibitor, inhibits the growth of cells expressing BCR-ABL, TEL-ABL, and TELPDGFR fusion proteins. Blood. 1997;90(12):4947-52.

7. Muller AM, et al. Imatinib mesylate as a novel treatment option for hypereosinophilic syndrome: two case reports and a comprehensive review of the literature. Ann Hematol. 2006;85 (1):1-16.

8. Schaller JL, Burkland GA. Case report: rapid and complete control of idiopathic hypereosinophilia with imatinib mesylate. MedGenMed. 2001;3(5):9.

9. Baccarani M, et al. The efficacy of imatinib mesylate in patients with FIP1L1-PDGFRalpha-positive hypereosinophilic syndrome. Results of a multicenter prospective study. Haematologica. 2007; 92(9):1173-9.

10. Bain BJ, Fletcher SH, Chronic eosinophilic leukemias and the myeloproliferative variant of the hypereosinophilic syndrome. Immunol Allergy Clin North Am. 2007;27(3):377-88.

11. Metzgeroth G, et al. Safety and efficacy of imatinib in chronic eosinophilic leukaemia and hypereosinophilic syndrome: a phaseII study. Br J Haematol. 2008;143(5):707-15.

12. Tefferi A. Blood eosinophilia: a new paradigm in disease classification, diagnosis, and treatment. Mayo Clin Proc. 2005; 80(1):75-83.

13. Fletcher S, Bain B, Diagnosis and treatment of hypereosinophilic syndromes. Curr Opin Hematol. 2007;14(1):37-42.

14. Pardanani A, et al. FIP1L1-PDGFRA fusion: prevalence and clinicopathologic correlates in 89 consecutive patients with moderate to severe eosinophilia. Blood. 2004;104(10):3038-45.

15. Weller PF, Bubley GJ. The idiopathic hypereosinophilic syndrome. Blood. 1994;83(10):2759-79.

16. Helbig G, et al. The results of imatinib therapy for patients with primary eosinophilic disorders. Eur J Haematol. 2006;76(6): $535-6$.

Avaliação: Editor e dois revisores externos Conflito de interesse: sem conflito de interesse

Recebido: 22/06/2009

Aceito: 20/07/2009 